\title{
Tangible Interaction on Tabletops for Elderly People
}

\author{
Tiago Marques ${ }^{1,2}$, Francisco Nunes ${ }^{1}$, Paula Silva ${ }^{1}$, and Rui Rodrigues ${ }^{2}$ \\ ${ }^{1}$ Associação Fraunhofer Portugal Research, EDIFICIO CENTRAL Rua Alfredo Allen \\ 455/461, 4200-135 Porto, Portugal \\ \{tiago.marques, francisco.nunes, paula.silva\} @fraunhofer.pt \\ ${ }^{2}$ Dept. Engenharia Informática - Faculdade de Engenharia da Universidade do Porto \\ Rua Dr. Roberto Frias s/n, 4200-465 Porto, Portugal \\ \{ei06076,rui.rodrigues\} @fe.up.pt
}

\begin{abstract}
The urge to improve the life of older adults grows as this segment of society expands. Computers have an enormous potential to benefit the lives of older adults, however, the unawareness or disregard of their characteristics, renders technology, many times, impossible to use. Peripherals are a common obstacle when learning to operate computers, because the most common ones do not directly map the input in the user interface. It has been argued that touchand gesture-based user interfaces, due to their direct mapping of input, can reduce the obstacles that older adults face, when using the computer. To assess this, this paper presents a project that uses a multi-touch tabletop system as a gaming platform for older adults. Specifically, it reports on the low-fidelity prototype that was built to test whether tangible objects can be used. Conclusions regarding the viability of tangible objects for that purpose are also drawn.
\end{abstract}

Keywords: Tangible objects, Tabletop, Games for elderly, Natural User Interface, Human-Computer Interaction.

\section{Introduction}

Population is getting older at an accelerated pace and should reach historical levels in the next years. Recent projections foresee that by 2060, 30\% of the European population will be 65 years or older [1]. This demographic change is shaping a new society with fewer young people and an ever-growing number of older adults [2]. This setting urges one to reflect on the needs of elders, in order to discover how to improve their quality of life in a society built for younger people.

The aging process is responsible for changes in the perception, cognitive and motor systems [3]. Nevertheless, if properly stimulated, it is possible to slow down or decrease the effects of certain changes in these systems. It is therefore relevant to create stimulation tools that are adequate for older adults.

Moreover, studies $[4,5,6]$ have shown that digital games can help maintaining and improving cognitive abilities, psychomotor skills, and social interaction.

The project that backdrops this paper, consists of a tabletop gaming system that aims to stimulate both the cognitive and motor systems of older adults. The specificities of this target audience, led one to devote special care into the design of 
the user interface, and on creating a system that interfered as little as possible with older adults' habits and traditions. This paper focuses on tangible objects used on tabletops and how they can be used to stimulate older adults.

\section{The Tabletop as a Platform for Older Adults to Play games}

Despite the growing number of older adults, technology is still designed for younger users. Even though older adults are receptive to technology, in most situations it is avoided because it is either not understood or because it ignores the characteristics of older adults and is therefore extremely difficult to use [7].

Natural User Interfaces (NUI) consist of interfaces that rely on natural interaction [8] to offer a direct mapping between the input device and the application. Touchbased technologies and NUIs may be presented as replacement for the keyboard and mouse as the computer's main input without having a significant learning curve associated [9].

The tabletop, a table sized touch-based user interface, can remove the barrier that keeps older adults from starting to use computers $[10,11,12]$.

Apart from the advantages offered by these user interfaces, tabletops can be used by multiple users, enabling collaborative work or collaborative games.

This project was aimed at understanding how tangible objects can be used to improve the quality of life of older adults by providing a better and richer experience when in contact with digital games and by enabling the user to interact with the application using real objects. In order to do it, the authors decided to observe how older adults interacted with the tabletop and how tangible objects could be used as opposed to using images displayed on the tabletop's surface. A low-fidelity prototype to enable these observations was made.

\section{Game Prototype}

This prototype aimed to understand how the elders interacted with the table while playing the game and to see whether tangible objects could be used in the game, and if so, how and in which situations. The prototype was based on the Air Hockey game [13] and consists of throwing a puck to the other player's goal, as shown in Fig. 1.
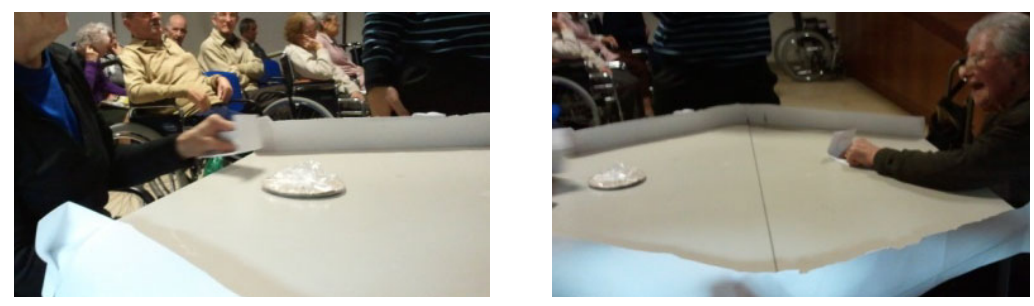

Fig. 1. Participants of the usability test playing with the game prototype 
This type of game should benefit older adults, as it requires a certain level of cognitive reasoning to deal with the reflection of the moving object when it hits the edges of the table and gross motor skills to interact with said object using hands or a tangible object.

Older adults felt an increasing motivation while playing the game. During the game, players were asked to use a tangible object instead of their hands. As a result, users tended to respect the game rules. By contrast, when not using the tangible object, users used both arms and tended to break the rules more often.

\section{Conclusions}

The observations showed that tangible objects cannot be used efficiently and with precision when the players actively use them. However, they have shown that tangible objects could be used to enforce the rules of the game. Moreover, players who had a greater motor decline were more prone to moving their arm energetically without being told to. This indicates that the use of tangible objects is likely to promote gross motor skills stimulation.

On the other hand, this test indicated that tangible objects should be seen as a proof-by-possession type of object rather than a gameplay type, as their usage on devices that have dead areas or poor object motion tracking may cause an unsatisfactory user experience.

\section{Future Work}

The studies conducted and described in this paper indicate that tangible objects may be a viable option to stimulate cognitive function and motor skills on elderly people. Further conclusions could be drawn if a high-fidelity prototype was used with the final users.

Different prototypes and ideas should also be tested, such as variations of the game, or even different types altogether. A high-fidelity prototype is planned and will integrate the results gathered through software testing on the tabletop and of prototype testing with the elderly people.

\section{References}

1. Eurostat (2011), http: / / epp. eurostat.ec.europa.eu/portal/ page/portal/eurostat/home/

2. Schulz, J.H., Binstock, J.H.: Aging nation: The economics and politics of growing older in America. Praeger, Westport (2006)

3. Fausset, C.B., Kelly, A.J., Rogers, W.A., Fisk, A.D.: Toward an understanding of and solutions for home maintenance and management needs for successful aging in place. Gerontologist 49, 393-394 (2009)

4. Fabregat, M.: Development of high therapeutic value ist-based games for monitoring and improving the quality of the life of elderly people. Information Society Technologies project CN 034552 (2006) 
5. Goldstein, J., Cajkp, L., Oosterbroek, M., Michielsen, M., Van Houten, O., Salverda, F.: Video games and the elderly. Social Behavior and Personality: an International Journal 25(4), 345-352 (1997)

6. Green, C.S., Bavelier, D.: The Cognitive Neuroscience of Video Games (2004)

7. Greengard, S.: Facing an age-old problem. Commun. ACM 52, 20-22 (2009)

8. Lee, J.C.: In search of a natural gesture. XRDS 16, 9-12 (2010)

9. Kjeldsen, R., Kender, J.: Toward the use of gesture in traditional user interfaces. In: Proceedings of FG 1996, vol. 136. IEEE Computer Society, Washington, DC, USA (1996)

10. Czaja, S.J.: Aging and the acquisition of computer skills. In: Aging and Skilled Performance: Advances in Theory and Application, pp. 201-220 (1996)

11. Czaja, S.J.: Computer technology and the older adult. In: Handbook of Human-Computer Interaction, 2nd edn., pp. 78-812 (1997)

12. Hollinworth, N.: Improving computer interaction for older adults. ACM SIGACCESS Accessibility and Computing 93 (2009)

13. Wikipedia, Air Hockey (2011), http: / / en.wikipedia.org/wiki/Air_hockey 